Licenciada en Educación Primaria por la Benemérita y Centenaria Escuela Normal del estado de Chihuahua. Máster en Desarrollo Educativo por el Centro Chihuahuense de Estudios de Posgrado. Actualmente doctorante en el Instituto de Pedagogía Crítica.

Historial editorial Recepción: 24 de septiembre de 2020.

Revisión: 17 de octubre de 2020. Aceptación: 28 de noviembre de 2020. Publicación: 13 de diciembre de 2020. 


\title{
Redes sociales: ¿Amenaza para los opresores o herramienta para oprimir?
}

\author{
Social Media: Threat To Oppressors \\ Or Tool To Oppress?
}

\section{Redes sociais: Ameaça aos opressores ou ferramenta para oprimir?}

\author{
Perla Marina Núñez Barrón \\ Instituto de Pedagogía Crítica \\ marinanub@hotmail.com
}

\section{Resumen}

Las redes sociales son una herramienta de comunicación que cada vez se vuelve más importante en la vida cotidiana de las personas, estas tienen diversos usos, entre ellos interactuar, comunicar y opinar sobre cualquier asunto, ese flujo de información puede ser utilizado para muchos fines. Uno de ellos sería liberar a las masas, al favorecer el análisis, la reflexión y el pensamiento crítico para generar conocimiento, información y participación de los usuarios en la vida democrática del país y en busca de una mejor calidad de vida. O su contraparte el uso de las redes sociales para oprimir, pues a pesar de la facilidad con que obtenemos la información ésta a veces llega manipulada, censurada o tergiversada debido a la vigilancia masiva que ejercen los opresores en internet. Ambos casos serán analizados a lo largo del presente artículo.

Palabras clave: Redes sociales, Movimientos sociales, Herramientas de opresión, Amenaza de opresores, Oprimidos. 


\section{Abstract}

Social networks are a communication tool that is becoming more and more important in people's daily lives, they have various uses, including interacting, communicating and giving an opinion on any matter, this flow of information can be used for many purposes. One of them would be to liberate the masses, by favoring analysis, reflection and critical thinking to generate knowledge, information and participation of users in the democratic life of the country and in search of a better quality of life. Or its counterpart, the use of social networks to oppress, because despite the ease with which we obtain information, it sometimes arrives manipulated, censored or misrepresented due to the massive surveillance exercised by the oppressors on the internet. Both cases will be analyzed throughout this article.

\section{Keywords: Social Networks, Social Movements, Tools of Oppres- sion, Threat of Oppressors, Oppressed.}

\section{RESUMO}

As redes sociais são uma ferramenta de comunicação que está a tornar-se cada vez mais importante no quotidiano das pessoas, têm várias utilizações, nomeadamente interagir, comunicar e opinar sobre qualquer assunto, este fluxo de informação pode ser utilizado para diversos fins. Uma delas seria libertar as massas, favorecendo a análise, a reflexão e o pensamento crítico para gerar conhecimento, informação e participação dos usuários na vida democrática do país e em busca de uma melhor qualidade de vida. Ou sua contrapartida, o uso das redes sociais para oprimir, porque apesar da facilidade com que obtemos informações, às vezes chega manipulada, censurada ou deturpada devido à vigilância massiva exercida pelos opressores na internet. Ambos os casos serão analisados ao longo deste artigo.

\section{Palavras-chave: Redes sociais, Movimentos sociais, Ferramentas de opressão, Ameaça de opressores, Oprimidos.}




\section{INTRODUCCIÓN}

Los humanos poseemos metas y objetivos propios, organizamos nuestra vida en grupos sociales de diferentes tipos, con distintos fines y características, pertenecemos a una familia, a un grupo de amigos, compañeros de escuela; así como el tránsito diario con personas que desconocemos, pero que voluntaria o involuntariamente influyen en nuestro modo de actuar.

"El hombre por naturaleza es un ser social. Desde que nace requiere del contacto y de la interacción de las personas para poder satisfacer sus necesidades tanto materiales como espirituales". (Borja, p. 39)

Actualmente (octubre 2020) esas interacciones también se realizan a través de redes sociales en internet - World Wide Web- que permiten el contacto con una gran cantidad de personas con intereses comunes (amistad, parentesco, gustos, aficiones, empleo, pasatiempos etc...) y lo que en un principio eran aplicaciones para divertir, comunicar e intercambiar información, actualmente tienen otros usos, en este texto focalizamos el oprimir o liberar masas a través de ellas.

La expansión abrumadora de estas redes permite expresar opiniones, juicios y críticas de diversos temas, además de servir como medio para la convocatoria de manifestaciones y protestas públicas con un mayor número de receptores, que en su mejor versión permitirían la liberación de los oprimidos, no obstante "Las redes sociales, enmarcadas en lo que hoy en día son las TICs (Tecnologías de la Información y las Comunicaciones) son uno de los tantos fenómenos que caracterizan a la posmodernidad.”( Elizondo, M \& Picot, p. 157),

Irónicamente también podemos observar que esta plataforma se ha convertido en una herramienta para controlar masas a través de la ejecución de una violencia simbólica (reforzando estereotipos, segregando, visibilizando a las minorías etc.) a veces sutil y en ocasiones muy contundente.

Ambos casos los ejemplificaremos a lo largo del presente ensayo para que el lector pueda responder con juicio propio a la pregunta génesis de este documento: Redes sociales ¿Amenaza para los opresores, o herramienta para oprimir?

\section{AMENAZA PARA OPRESORES}

Para los años 20 del siglo Xxi, en las redes sociales se genera un importante tráfico de información con una audiencia elevada, tan solo Facebook (la red social más usada por los mexicanos) 2.449 millones 
de usuarios en el mundo (Galeano, p.1) quienes están en contacto con individuos con los que comparten intereses, inquietudes y en algunos casos deseos de transformar su situación actual, encontrando en estos la fuerza para actuar.

Por lo anterior, Internet se ha convertido en una terrible amenaza para los gobiernos opresores por considerarlo un instrumento emancipador, la principal amenaza es el poder que tiene como medio de convocatoria para manifestaciones públicas ya que favorece la organización, coordinación y toma de decisiones en proyectos de transformación social.

Una herramienta que utiliza este tipo de convocatorias es el término hashtag (\#) el cual es utilizado para etiquetar o clasificar publicaciones de acuerdo a un tema o contenido; en lo que se refiere a los movimientos sociales, este, ha sido utilizado para la difusión de los mismos logrando así trascendencia internacional.

Para ejemplificar esta situación, en México, se gestaron algunos movimientos sociales que utilizaron como medio la red informática mundial, tal es el caso del Ejercito Zapatista de Liberación $\mathrm{Na}$ cional (EZLN), el movimiento \#YoSoy132, el matrimonio igualitario o el aborto que son motivo de debates, marchas y protestas de quienes exigen derechos y de quienes se oponen a esto. A continuación, los describiremos brevemente.

El movimiento Zapatista tuvo su génesis en la inconformidad de los indígenas campesinos del estado de Chiapas, México, ante el sistema político mexicano y sus promesas de modernidad, por lo que estaban en contra de la globalización neoliberal y el Tratado de Libre Comercio de América del Norte.

Aunque los inicios del movimiento del EZLN no fueron en las redes sociales, fue el primer movimiento que hizo uso de la tecnología e informática, la primera página web para dar a conocer los comunicados del grupo armado a través del ciberespacio (www. ezln.org.mx.) fue creada por un estudiante de Texas en 2005 logrando atención mediática y trascendencia internacional, así como el apoyo de miembros de organizaciones no gubernamentales e intelectuales de Latinoamérica y Europa.

$\mathrm{Al}$ respecto Zaragoza (p. 1) expresa que "Es notable lo que el avance tecnológico pudo hacer por el movimiento zapatista: llevarlo de la selva chiapaneca, en el olvidado sureste mexicano, al mundo entero, a la solidaridad de la intelectualidad europea, latinoamericana, estadounidense y, por supuesto, la nacional". 


\section{E1 movimiento \#YoSoy132}

Este movimiento utilizó dos protestas paralelamente una presencial y la otra virtual. La presencial se originó cuando jóvenes estudiantes de la Universidad Iberoamericana cuestionaron los excesos de seguridad del entonces gobernador del estado de México, Enrique Pena Nieto, en su visita a la universidad, además de críticas a su desempeño como funcionario. En consecuencia, políticos del Partido Revolucionario Institucional los acusaron de no ser estudiantes de la institución ya mencionada, por lo que los pupilos realizaron un video donde mostraron sus credenciales y lo denominaron 131 estudiantes responden es aquí donde surge la segunda protesta, denominada \#YoSoy 132.

Esta protesta toma su nombre cuando se empezaron a auto afiliar estudiantes de universidades públicas y privadas de todo el país, con el hashtag \#YoSoy132 haciendo alusión a que eran un estudiante más que exigían un uso democrático de los medios de comunicación, para evitar la imposición mediática de Enrique Peña Nieto como candidato en las elecciones presidenciales 2012 de México.

En este movimiento se pudo observar el potencial político de internet al respecto Rivera (párr.9) expresa que:

Las primeras estrategias comunicativas empleadas por \#YoSoy132 pusieron a prueba la magnitud emancipadora de internet, "tal vez sea más exacto sugerir que internet ofrece a las personas comunes el potencial de este poder" (Tang y Yang, 2011 citado en Rivera). \#YoSoy132 se apoyó en la Web 2.0 para convocar a marchas con el fin de impulsar su primera demanda: la democratización del sistema de medios.

Otros movimientos que fueron tendencia en el país con el uso de hashtag cómo \#AyotzinapaSomosTodos, el cual exigía respuesta respecto al paradero de 43 estudiantes normalistas \#MeTooMexicano, quien pretendía visibilizar el acoso laboral o la de la lucha por los derechos de los de integrantes de la comunidad LGBTQ (acrónimo de lesbiana, gay, bisexual, transgénero y queer) estos hicieron uso del hashtag \#AmorEsAmor, para dar mayor difusión a sus demandas, entre las que destacan el matrimonio igualitario y la penalización de los esfuerzos para corregir la orientación sexual, pro-vida y su contraparte pro-aborto, han encontrado en las redes sociales una plataforma para sus campañas aunque no se han resuelto en su totalidad han mostrado avances.

Zaragoza (párr.23) expresa que:

Esto nos muestra que la Internet no soluciona por sí misma los problemas, no es la panacea tecnológica. En síntesis, la reunión de personas con intereses mutuos no comenzó, de modo alguno, con los sitios en la world wide web. Pero, sin lugar a dudas, la informática ha dado mayores posibilidades 
a aquellos que así lo buscan... La tecnología por sí misma no resuelve el problema, pero promueve o plantea soluciones.

Pero en este senito Rivera (párr.6) nos alerta sobre la contraparte del uso de la tecnología:

El ciber-utopianismo, que es la creencia naive de que las ventajas proporcionadas por las nuevas tecnologías van a ser utilizadas tan sólo en sus aspectos positivos, por parte de ciudadanos y activistas por la democracia, sin tener en cuenta la contrapartida, que en ocasiones es incluso peor que las ventajas que nos ofrecen.

En el siguiente apartado hablamos del lado oscuro de las redes sociales aquel que es usado como herramienta de opresión.

\section{Herramientas para oprimir}

Las redes sociales también tienen aspectos que atentan contra la libertad e intimidad de los usuarios y la mayoría de las veces, son ellos mismos quienes lo permiten debido a la gran cantidad de información que comparten de manera consciente o inconsciente, información que puede ser utilizada como instrumento de opresión.

Asimismo, las redes sociales pueden servir para mantener "anestesiados" a los usuarios ya sea manipulando, censurando o creando falsa información para conservar el orden social que les favorece a los opresores.

Este apartado se organizó con algunas categorías propuestas por Freire (2005) contrastándolas con las nuevas formas de control social.

\section{La ciencia como instrumento para sus finalidades}

En este apartado hablaremos de la ciencia de la información por ser la más ligada al uso de las redes sociales ya que tiene como objeto de estudio la información en un contexto tecnológico Taylor (citado en Munera, p.58) la conceptualiza de la siguiente manera:

La ciencia que investiga las propiedades y el comportamiento de la información, las fuerzas que gobiernan su flujo y los medios para procesarlas para su acceso y uso óptimo. El proceso incluye la generación, diseminación, recolección, organización, almacenamiento, recuperación, interpretación y uso de la información. 
Esta ciencia y el desarrollo tecnológico se combinan para desarrollar una estrategia de vigilancia a los usuarios y sus interacciones de una forma masiva, en esta vigilancia participan empresas para que consuman sus productos y el gobierno como forma de control social.

García (p.1) hace una comparación de la sociedad panóptica de Foucault y la vigilancia masiva que ejercen los gobiernos en internet "donde el modelo de control social comenzó siendo una trágica historia de pérdida de libertad e intimidad, aceptada voluntaria y gustosamente por los sujetos" tal y como se vive actualmente en las redes sociales.

Prueba de esto es la denuncia de Julián Assange, fundador, editor y portavoz del sitio web WikiLeaks en 2011 acusando a grandes corporaciones de vigilar la información de cada persona a través de sus celulares, correos electrónicos y redes sociales para después ponerla a la venta al mejor postor o lo que hizo Edward Snowden un trabajador de la Agencia Central de Inteligencia cuando en 2013 público pruebas que las agencias de seguridad estatales utilizaban la vigilancia masiva para recoger y analizar millones de comunicaciones privadas de personas en todo el mundo,

Contrariamente al auge digital donde deberíamos estar más y mejor informados una sociedad vigilada es susceptible a recibir información manipulada, censurada o tergiversada de conflictos actuales, por ejemplo en redes sociales el tema destacado es que la hija de un cantante famoso no quiera entregar el cuerpo a sus medios hermanos, mientras en el país aquejan problemas la desaparición forzada de miles de personas, el conflicto del agua en Chihuahua donde campesinos han protestado para impedir que las presas de la entidad entreguen agua a Estados Unidos, la inminente crisis económica a raíz de la pandemia, entre otros no menos importantes problemas.

Esto queda expresado en la ilustración 1 dando similar importancia a la situación ya descrita donde Sara Sosa ${ }^{1}$ no quiere entregar el cuerpo de su famoso padre y lo comparan con la circunstancia vivida en Ayotzinapa donde desaparecieron 43 estudiantes normalistas donde ambas situaciones quedan como temas banales por la falta de análisis y reflexión del mensaje.

1 Ver lustración 1: hija de José José, cantante mexicano 


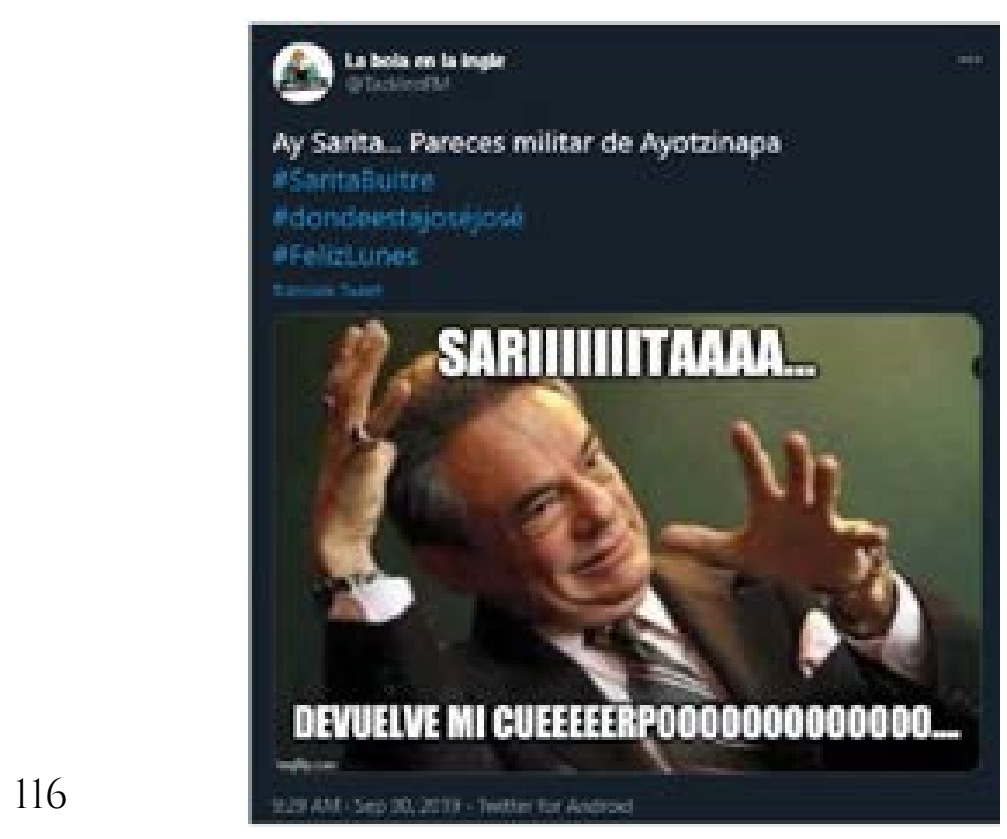

Ilustración 1. Memes de Sarita fuente: twitter usuario la bola en la ingle

Asimismo, la vigilancia de aquellos que representan una amenaza para los opresores permite conocer la información que comparten para asegurarse de censurar antes de que llegue a más audiencia, así como difundir propaganda desacreditante de los mismos y de esta manera conservar el orden social que les favorece. "La vigilancia será la estrategia que reemplace progresivamente a la coerción física como un medio para mantener el orden y la armonía de los individuos". (García, p.2)

Las personas ya no son libres en la red, utilizan sus dispositivos electrónicos para "navegar" en los mundos que han creado para ellos y gracias a ellos, debido a la gran cantidad de información que otorgan de manera consientes o inconsciente. En relación a esto Luna (citado en Estrada, párr. 1) expresa que: "Google no es tu mejor amigo ni puede leer tu mente. Sin embargo, lo que sí puede hacer, es llenarte de cookies para estudiar tu comportamiento como una rata de laboratorio "

En este sentido Estrada (párr. 2) resalta lo inquietante de "la falta de privacidad en el mundo actual, donde los gobiernos y corporaciones nos vigilan, además de que utilizan la información que ponemos en las redes sociales, sin que muchos lo sepamos ni estemos conscientes de ello". 
Varios ejemplos de vigilancia del gobierno los podemos enumerar con base en la investigación realizada por Damano (2018), aquí se presenta los puntos más importantes:

1. En Cuba 41 sitios fueron bloqueados por expresar críticas al gobierno, informar sobre cuestiones de derechos humanos o abordar técnicas para esquivar la censura.

2. En Francia, España y Reino Unido, entre otros países, se utilizaron leyes de ambigua definición que penalizaban el "enaltecimiento" o la "apología" del terrorismo para procesar a activistas y grupos de la sociedad civil por opiniones expresadas en Internet y en redes sociales.

3. Las autoridades de Tailandia presionaron a Facebook, Google y YouTube para que retiraran contenidos de Internet, incluidos contenidos que se consideraban críticos con la monarquía.

4. En India, las autoridades cerraban con frecuencia servicios de Internet, alegando motivos de orden público.

5. En Egipto detuvieron al menos a 240 activistas políticos y manifestantes por cargos relacionados con publicaciones en Internet que las autoridades consideraron "insultantes" para el presidente o por participar en protestas no autorizadas.

6. El Estado de Palestina adoptó en julio la Ley de Delitos Electrónicos, que permitía la detención arbitraria de periodistas, denunciantes de irregularidades y otras personas que criticaran a las autoridades en Internet.

7. Essam Koshak fue citado para ser interrogado por las autoridades de Arabia Saudí; le preguntaron de forma insistente sobre su cuenta de Twitter. Se enfrentaba a varios cargos relacionados con su activismo en Internet.

8. En Pakistán se sometió a desaparición forzada a cinco blogueros críticos con el gobierno y se detuvo a otros por haber hecho comentarios críticos con el ejército o haber expresado ideas consideradas "contrarias al islam".

9. Las autoridades de Kazajistán que ya habían conseguido asfixiar a los medios de comunicación independientes, emplearon métodos cada vez más sofisticados y agresivos para erradicar las voces disidentes en Internet y las redes sociales.

10. En Azerbaiyán se puso en marcha una cibercampaña específica contra las voces críticas. 


\section{Visión inauténtica del mundo}

Actualmente, los mexicanos pasamos casi el 40\% de nuestro tiempo conectados en alguna red social (según la Asociación Mexicana de Internet) demostrando así el importante papel que juegan en la vida cotidiana de las personas.

Estas "interacciones" sociales nos han convertido en individualistas que miran más a una pantalla de un dispositivo electrónico que a los ojos de sus semejantes, se puede reaccionar a las publicaciones, pero al verse en vivo evitan el contacto, viviendo entonces en un mundo de apariencias y de falsas amistades.

En lo que corresponde a movimientos sociales se protesta virtualmente, se firman peticiones online, se aceptan invitaciones a manifestarse, se publican opiniones o se responde a opiniones de otro, se posicionan de un lado o de otro en temas radicales, pero de ahí no pasa, no se materializa en acciones, no se reflexiona a profundidad por lo tanto no se cierra el círculo para ser una praxis.

Nos referimos entonces como visión inauténtica en redes a la incongruencia entre la gran actividad en internet y la poca o nula participación presencial en protestas o propuestas en torno a la defensa de los derechos humanos y sociales.

\section{Conocimiento de sí mismos, como oprimidos}

Giroux expresa que los oprimidos participan activamente en su sometimiento esto se observa cuando los usuarios de redes sociales entregan su privacidad solo por formar parte de estas plataformas, cada "like" (simbolismo de internet que permite al usuario dar un retroalimentación positivo a cualquier tipo de contenido), visita, búsqueda o contactos frecuentes dan información que permite a los opresores controlar y disciplinar según su conveniencia.

El estudio, publicado en la revista Proceedings of the National Academy of Sciences y liderado por el investigador Youyou Wu, encontró que con 10 'me gusta', una máquina puede conocer a alguien mejor que un compañero de trabajo; con 70, mejor que su compañero de cuarto; con 150, mejor que un familiar, y con 300, mejor que su propia pareja. (Patiño, párr. 3)

Como ejemplo del conocimiento de sí mismos como oprimidos está el uso de memes (Texto, imagen o vídeo difundido en internet con fines humorísticos) donde con la bandera de comedia incorporan a su subconsciente su conformidad con esta posición y creando vínculos de dependencia con sus opresores; tal es el caso de los denominados "Godínez" donde hacen burla de sí mismos al sen- 
tirse inferiores al jefe o al esperar el viernes de quincena como única meta, otros más donde mujeres se sienten orgullosas de ser "toxicas" y sus parejas naturalizan la situación como algo cómico, o aquellos donde mujeres se avergüenzan de ser "mamá luchona" etiqueta que les han dado los usuarios de redes sociales a las madres solteras que no encajan en el estereotipo de madre abnegada, pero son ellas mismas, las que comparten orgullosas estas publicaciones; otra muestra son los memes donde los hombres de avanzada edad con una buena posición económica adquieren estatus de "Sugar Daddy" al salir con chicas menores y donde ambos sienten que utilizan al otro.

"Su dependencia emocional, fruto de la situación concreta de dominación en que se encuentran y que, a la vez, genera su visión inauténtica del mundo, no puede ser aprovechada a menos que lo sea por el opresor. Es éste quien utiliza la dependencia para crear una dependencia cada vez mayor" Freire (en Giroux, p.67)

\section{Conclusiones}

La máquina la hace el hombre, y es lo que el hombre hace con ella lo que define su utilidad, ya sea para bien o para mal Jorge Drexler

Las redes sociales por sí solas no son ni amenaza para los opresores ni herramientas para oprimir, todo depende del usuario, si esté es un pensador crítico radical, la amenaza para los opresores es inminente, ya que estas permiten potencializar la libertad de expresión, superar fronteras físicas y culturales, una mayor difusión y en el mejor escenario lograr la transición de información a conocimiento, razón y participación.

Si quien manipula las redes sociales es un opresor recurrirá a su poder para seguir disfrutando su posición, por ejemplo, el gobierno de Cuba que cerró todos aquellos sitios web que criticaran el gobierno, las grandes empresas que extraen la información de nuestras navegaciones para favorecer el consumismo y/o mantener anestesiado al oprimido, en un falso mundo de felicidad que ha creado el opresor para él.

Las redes sociales utilizan una opresión difícil de detectar por la parte afectada mientras no saturan de todo aquello que nos gusta, censuran todo lo que pueda liberarnos, pero como estamos ocupados con lo primero no reflexionamos sobre lo segundo. 
Pero si algún oprimido logra esta reflexión puede no recurrir al uso de las tecnologías para conservar su libertad o aprovechar las oportunidades que estas ponen a su disposición para emanciparse.

\section{REFERENCIAS}

Borjas M. La evaluación del aprendizaje como compromiso: una visión desde la pedagogía crítica. 2014 impreso. México.

Damano D. En plena era digital, internet continúa siendo una temida amenaza para gobiernos opresores. Blog Amnistía Internacional. 2018. Blog Amnistía Internacional octubre 2020 https://www.cva.itesm. $\mathrm{mx} /$ biblioteca/pagina_con_formato_version_oct/apaweb.html.

Elizondo, M \& Picot, C. El sujeto posmoderno en las redes sociales., de FaHCE 2011 , septiembre 2020 Sitio web: http://www.memoria. fahce.unlp.edu.ar/trab_eventos/ev.1539/ev.1539.pdf

Estrada R.. 'Privacidad': el lado oscuro de las redes sociales., de El financiero, 2018 octubre 2020 Sitio web: https://www.cva.itesm.mx/biblioteca/pagina_con_formato_version_oct/apaweb.html

Freire P. Pedagogía del Oprimido.55 ed.; Siglo XXI, 2005, editores. México.

Galeano S. "Cuáles son las redes sociales con más usuarios del mundo (2020).” marketing 4 Ecommerce3 febrero 2020: 1. marketing 4 Ecommerce. 22 octubre 2020 https://marketing4ecommerce. net/cuales-redes-sociales-con-mas-usuarios-mundo-2019-to$\mathrm{p} / \#: \sim:$ text=E1\%20informe $\% 20$ mostraba\%20que $\% 20$ en,un $\% 20$ $7 \% 2 \mathrm{C} 8 \% 25 \% 20 \mathrm{~m} \% \mathrm{C} 3 \% \mathrm{~A} 1 \mathrm{~s}$.

García Jiménez, R. Panoptismo: nuevas formas de control social, 2009 , en Contribuciones a las Ciencias Sociales, noviembre 2019 recuperado en www.eumed.net/rev/cccss/06/rgj2.htm

Giroux H. Teoría y resistencia en educación. Una pedagogía para la oposición. Prologo Paulo Freire. 2004, Siglo XXI editores. México DF

Munera M. CiENCIA DE LA INFORMACión Y GESTIÓN DEL CONOCIMIENTO, 2012, octubre 2020, de Dialnet Sitio web: https://www.cva.itesm.mx/biblioteca/pagina_con_formato_version_oct/apaweb.html

Patiño L.¿Cuánto valor tiene un 'like’ en su vida?. 2019,octubre 2020, de Eltiempo Sitio web: https://www.eltiempo.com/tecnosfera/novedades-tecnologia/el-significado-de-un-like-en-las-redes-sociales- 406570 
Rivera J. Evgeny Morozov: El Engaño de la Red, cómo no liberar el mundo.2011, septiembre 2020, de Sociología y Redes sociales Sitio web: http://sociologiayredessociales.com/2011/12/evgeny-morozov-elengano-de-la-red-como-no-liberar-el-mundo/

Rivera R. De la Red a las calles: \#YoSoy132 y la búsqueda de un imaginario político alternativo. 2014, octubre 2020, de scielo Sitio web: http://www.scielo.org.mx/scielo.php?script=sci_arttext\&pi$\mathrm{d}=\mathrm{S} 0187-57952014000200004$

Zaragoza M. Zapatistas sin fronteras. Las redes de solidaridad con Chiapas y el altermundismo, 2010. octubre 2020, de Scielo Sitio web: http://www.scielo.org.mx/scielo.php?script=sci_arttext\&pi$\mathrm{d}=\mathrm{S} 1870-23332010000200009$ 